\title{
e-Phaïstos
}

e-Phaïstos

Revue d'histoire des techniques / Journal of the history

of technology

V-1 2016 | 2018

Ville et technique

\section{Forêt et industrie en Côte d'Ivoire de 1910 à 1980. Une histoire entre techniques et sociétés}

\section{Koffi Innocent Diezou}

\section{(2) OpenEdition}

Journals

Édition électronique

URL : http://journals.openedition.org/ephaistos/1192

DOI : 10.4000/ephaistos. 1192

ISSN : 2552-0741

Éditeur

IHMC - Institut d'histoire moderne et contemporaine (UMR 8066)

\section{Référence électronique}

Koffi Innocent Diezou, « Forêt et industrie en Côte d'Ivoire de 1910 à 1980. Une histoire entre techniques et sociétés », e-Phaïstos [En ligne], V-1 2016 | 2018, mis en ligne le 21 janvier 2018, consulté le 09 novembre 2019. URL : http://journals.openedition.org/ephaistos/1192

Ce document a été généré automatiquement le 9 novembre 2019.

Tous droits réservés 


\title{
Forêt et industrie en Côte d'Ivoire de 1910 à 1980. Une histoire entre techniques et sociétés
}

\author{
Koffi Innocent Diezou
}

Thèse : références bibliographiques

Koffi Innocent Diezou, Forêt et industrie en Côte d'Ivoire de 1910 à 1980. Une histoire entre techniques et sociétés, thèse de doctorat en Histoire de l'Université Paris 1 Panthéon-Sorbonne, ED 113, Centre d'Histoire des Techniques (IHMC, UMR 8066), soutenue le 02 décembre 2015.

\section{Directeur de thèse \\ Anne-Françoise GARÇON, Professeur, Université Paris 1 Panthéon-Sorbonne \\ Jury \\ André Guillerme, Professeur, Conservatoire national des arts et métiers (CNAM Paris) \\ Jean-François Belhoste, Directeur d'Études à l'École Pratique des Hautes Études (EPHE) \\ Hélène Timpoko-Kabore, Maître de conférence HDR, Université Félix Houphouët- Boigny, Abidjan, Côte d'Ivoire}

1 Monsieur le président, Mesdames et Messieurs les membres du jury, honorables invités, je suis honoré cet après-midi de présenter à votre auguste assemblée les résultats de ma thèse, étape indispensable à la noble carrière de scientifique et chercheur que je souhaiterais entreprendre dans l'avenir.

2 Avant toutes choses, je voudrais avec votre permission rappeler le thème de ma thèse : Forêt et industrie en Côte d'Ivoire de 1910 à 1980 : Une histoire entre techniques et sociétés. Ce thème tire ses origines d'un constat récurent, lorsque l'on se déplace en 
région sud-forestière de la Côte d'Ivoire. Il n'est pas rare que le regard soit attiré par un ensemble d'exploitations, de taille variable, modestes, austères, parfois caractérisées par une architecture particulière, souvent envahies par la végétation ou confondues dans le paysage. Ce qui attire le plus l'attention, c'est le fait qu'elles soient gardées, tantôt par des policiers ou gendarmes, tantôt par des particuliers. La curiosité s'attise du fait des clôtures, parfois grilles métalliques séparées par des pilots de ciment armé à intervalle régulière, parfois palissades de bois de scie. Lorsque l'on interroge les populations qui vivent aux abords, sur la nature de ces exploitations, c'est le silence, ou alors la réponse arrive invariable : "C'est la propriété du Blanc», souvent en le nommant, « Gantois, Eglin, Ernaud, Jacob... ».

3 Confronté au silence assourdissant des populations et à la présence d'appareils et de machines que l'on peut mettre aisément en relation avec des unités de production en série, il nous est apparu important de nous y intéresser à travers une étude scientifique à l'aune de nos acquis en histoire des techniques. La Côte d'Ivoire a connu dans son histoire une phase coloniale et post-coloniale. L'énoncé de ces noms n'est pas sans effets sur la mémoire de ces populations. À cela s'ajoute des survivances, des similitudes, des formes d'emprunt observables dans les exploitations fondées par les populations autochtones. On se retrouve ainsi confronté à des faits qui transgressent les deux périodes, coloniale et post-coloniale.

4 Les premiers contacts directs entre les populations ivoiriennes et européennes datent $\mathrm{du} \mathrm{XV}^{\mathrm{e}}$ siècle. Cependant les relations visant à l'exploitation des premières ressources au plan industriel tirent leur origine des rapports de mission La Malouine menées dans par le capitaine de Corvette Bouët-Willaumez en 1840. Il y dresse l'étalage des richesses importantes du territoire. Ce rapport a occasionné une seconde mission menée par le lieutenant de vaisseau Fleuriot de Langle en 1842 au cours de laquelle il signe des traités de protectorat entre la France et les populations de la côte sud-est de la Côte d'Ivoire. C'est donc le ministère de la marine qui a donné une impulsion décisive à l'entreprenariat industriel sur ce territoire qui est devenu cinquante années plus tard, la Côte d'Ivoire reconnue en tant que colonie française sous l'administration du lieutenant-gouverneur Binger.

Dans un premier temps, les Français intéressés par les richesses vantés par les explorateurs du ministère de la Marine et du Muséum d'Histoire naturelle observent des gestes, des habitudes, des modes opératoires propres aux Africains pour produire et exploiter leurs ressources. Il faut dans ce contexte précis noter que l'entreprise de production en Côte d'Ivoire n'a pas été une activité conçue pour elle-même au départ, $\mathrm{du}$ fait des tensions en France entre politiques, scientifiques, philosophes, et diverses associations dont l'une des plus actives fut l'association Colonies-Sciences. Le but assigné fut donc de générer et fournir à la métropole française des matières premières exploitables, bois pour les traverses de chemins de fer, huile pour les savonneries, à commencer par celles de Marseille..., etc.)

6 Il a fallu, pour y parvenir, inventer une organisation du travail et de la main-d'œuvre, et donc procéder à des innovations techniques et technologiques pour produire plus et mieux. Les limites sont apparues, en effet, aussitôt la mise en œuvre du projet d'exploitation. Le constat établi par le lieutenant-gouverneur Clozel, signale le faible peuplement de la une surface exploitable du point de vue industriel, le sud-forestier en l'occurrence. La crise de la main-d'œuvre survint donc rapidement, due, d'une part à sa rareté, et, d'autre part, à une compréhension de la relation au travail totalement 
opposée à celle de l'Européen. Il en résulta le passage, sous la force de la contrainte, de ce que j'ai interprété comme des complexes techniques traditionnels vers des complexes techniques mixtes. Cette contrainte s'est déclinée en plusieurs méthodes qui se sont toutes soldées par la ferme résolution de former la main-d'œuvre locale. Il fallut en plus, éduquer, re-éduquer et instruire des techniciens auxiliaires, en évitant soigneusement d'en faire des concurrents. Il a résulté de l'industrialisation des sites sur lesquelles résidaient les populations autochtones, outre la transformation des modes opératoires, l'instauration d'un nouveau type de rapports au progrès, au développement technique et à la conduite des activités.

7 Quel état des lieux peut-on en faire désormais ? L'industrie peut-elle constituer un patrimoine pour la Côte d'Ivoire, alors que l'ensemble du matériel industriel ou technique provient d'un transfert de savoir, avec des savoir-faire techniques européens (Français en particulier) excluant l'habileté de l'homme Africain? Comment peut-on se reconnaître dans un patrimoine industriel présent sur son territoire, dont on ne possède pas complètement la culture technique?

8 Je n'ai pas cherché, dans cette recherche, à conduire une étude spécifique sur le monde traditionnel africain-ivoirien. Il ne s'agit pas non plus de mener une étude centrée sur les outils. L'enjeu est d'étudier une période durant laquelle il y a eu croisement entre deux cultures techniques. Un croisement qui s'est avéré parfois conflictuel. Eu égard aux vestiges en place, composés de machines, de tapis roulants, abrités dans des hangars, des fermes, eu égard aussi à la présence des mêmes agencements techniques dans les ateliers de production actuels en Côte d'Ivoire, en une manière de répétition évocatrice d'une perpétuation des savoir-faire, mon étude vise à lever ce paradoxe d'une cohabitation de témoins matériels longtemps mêlés à l'environnement de vie local, avec le sentiment d'incompréhension quant à la manière et aux raisons de leur établissement.

9 Cette ambition m'a conduit à une relecture des sources, selon de nouveaux angles d'analyses en lien avec le contexte africain. J'ai mis à profit, pour ce fait, des acquis issus des séminaires en histoire des techniques, en patrimoine industriel et des études au Centre National des Arts et Métiers relatives au béton et de ses différents usages. Les travaux d'Hélène Vacher sur l'architecture coloniale dans le Maghreb constitue en complément une référence majeure pour ce qui concerne les territoires africains coloniaux ${ }^{1}$.

10 J'ai préalablement orienté mes recherches vers les Archives Nationales de France. Celles-ci, situées à Fontainebleau. Les fonds consultés à Fontainebleau concernaient les compagnies à capitaux à vocation industrielle qui s'étaient développées dans les années 1930. Cela ne correspondait pas véritablement à mes attentes. Mais cela m’a conforté dans mon ambition de m'intéresser aux industries pionnières. Et cela m'a procuré une première confrontation avec l'étude de la production industrielle. La consultation des archives de l'Académie des sciences d'outre-mer à Paris, m'a permis d'affiner mes recherches sur les premières ébauches d'une industrie dans les colonies de l'Afrique occidentale française. Elles contiennent en effet de nombreuses sources imprimées parfois de première main sur l'industrie du bois en démontrant clairement le rôle important des militaires du génie dans l'architecture des unités de production. Il s'agit principalement de la priorité qu'ils ont accordée à l'usage du bois en lieu et place du métal, et du choix du béton pour consolider les structures. Enfin, pour circonscrire mes investigations, il m'est apparu opportun de consulter les archives nationales de la Côte 
d'Ivoire. Cette quête m'a permis de rassembler des informations pertinentes, notamment dans les séries portant sur les affaires politiques et économiques. Les journaux Fraternité (quotidien ivoirien devenu aujourd'hui Fraternité Matin) et I.D (pour Idée magazine) ont achevé de m'orienter dans l'identification des sites et villes, pour décider des contours de l'étude de terrain qui me semblait indispensable.

11 Pour mener à bien cette étude de terrain il a fallu préciser mes interrogations, les recouper et les vérifier. J'ai procédé par des entretiens avec des autorités préfectorales des villes identifiées. Muni d'autorisations préfectorales, j'ai pu, à l'été 2010, analyser les sites de production industrielle couvrant le littoral sud-forestier et la moitié sud de la Côte d'Ivoire. Ces études ne se sont pas limitées aux sites industriels. Les infrastructures de communication et ouvrages d'art y ont été intégrés, ainsi qu'une étude poussée des matériaux de construction que sont principalement le bois et le béton armé. Cette étude de terrain aurait dû s'enrichir d'une seconde en 2011, qui devait porter sur les industries longeant le chemin de fer, « outil de drainage » selon les administrateurs coloniaux, dans la moitié nord du pays où l'on trouve des industries sucrières. L'avènement de la crise militaro-politique en Côte d'Ivoire a brutalement freiné cette étude qui allait certainement apporter des éléments nouveaux. Ce fait majeur m'a ramené aux études archivistiques. Aussi, ai-je consulté les fonds des archives d'outre-mer d'Aix en Provence, portant sur les différents rapports des administrateurs coloniaux et officiers militaires relatifs aux comportements réfractaires des populations sur l'acquittement de l'impôt et le travail réglé. En dépouillant ces archives, je me suis aussi penché sur l'étude des infrastructures de développement antérieurement abordées aux archives nationales de la Côte d'Ivoire. Ces fonds beaucoup plus riches m'ont permis de développer une approche nouvelle des infrastructures routières. Non seulement la route crée la ville, mais elle consolide aussi l'industrie et accroît l'industrialisation en désenclavant les lieux de production.

12 Au delà de cette lecture des sources, des enquêtes orales ont été menées auprès des populations vivant sur les sites identifiés dans les villes qui ont fait l'objet de mon étude de terrain. Il est clairement apparu que ces personnes sont des descendants d'anciens ouvriers des Européens, et qu'elles ne savaient pratiquement pas grand-chose des activités qui se déroulaient dans les unités de production aujourd'hui fermées. Et pour celles en activité, à l'exception de leurs tâches dans la chaîne de production, elles ne pouvaient fournir des informations complémentaires. Les informations que les personnes m'ont fournies étaient dans une certaine mesure crédibles du fait de l'actualité nationale de la Côte d'Ivoire qui célébrait ses cinquante années d'indépendance. Dans cette dynamique, ces personnes ont exprimé une réelle motivation à fournir des informations, qui malgré leur importance avaient l'inconvénient d'être peu précises.

13 Cela a constitué un élément majeur dans les difficultés que renferme une telle étude. Surtout celle qui concerne le labeur des populations. La Côte d'Ivoire étant en crise, bon nombre des sites sont restés fermés pour des raisons juridiques opposant bien souvent l'État de Côte d'Ivoire à des particuliers. Le site Ernaud illustré par des prises de vue très éloignées, en pages annexes de ma thèse, en est une parfaite illustration. La fracture ou la césure entre le nord et sud de la Côte d'Ivoire a freiné une étape très importante de mon étude de terrain, puisqu'il s'agissait de la partie incluant certaines fabriques et/ou unités de production construites dans les régions du nord dont de nombreuses similitudes ou liens auraient pu être établis avec celles du sud. La rupture 
des financements et l'impossibilité d'en obtenir m'ont conduit à entreprendre une telle recherche sur fonds propres, bien insuffisants par ailleurs, pour mener à bien les multiples déplacements, que ce soit à Aix-en-Provence et en Côte d'Ivoire. C'est le lieu de saluer la solidarité et les échanges entre doctorants et chercheurs durant les différents séminaires.

Une relecture dépouillée des sources m'a conduit à les réinterpréter au vu des difficultés en me posant cette question : Quel patrimoine technique et industriel valoriser? Une tentative de réponse m'a conduit à privilégier les vestiges matériels dans un premier temps, et ensuite à m'orienter vers l'immatériel dans une seconde approche. Le matériel, ce sont essentiellement les hangars industriels, les machines, les outils. L'immatériel ce sont les discours qui les accompagnent, les bruits, les odeurs, la douleur ressentie par ces populations, les problèmes sanitaires qu'une médecine non complètement outillée ne parvient toujours pas à expliquer fondamentalement etc. J'ai privilégié le matériel, là où, dans mon enquête de terrain, le discours était quasi inexistant, et les souvenirs assez flous.

L'approche en termes de patrimoine industriel doit donner à voir ces unités et les espaces de production dans cette dualité. Espaces d'avenir et espaces en devenir, patrimoine de la douleur ou du rejet. C'est dans cette dualité que cette approche nouvelle typiquement contextuelle, du point de vue des études portant sur la production industrielle en Afrique s'inscrit. Il s'agira de fournir des instruments nouveaux aux populations pour comprendre leur territoire de production, mieux maitriser leurs acquis techniques afin de mieux mettre en valeur, ou encore mieux présenter leur identité industrielle spécifique qui les inscrira à long terme dans le processus du développement durable.

Monsieur le président, Mesdames et messieurs membres du jury, je vous remercie pour votre écoute.

\section{NOTES}

1. Vacher Hélène (dir.), Villes coloniales aux XIX ${ }^{e}-X X^{e}$ siècles. D'un sujet d'action à un objet d'histoire (Algérie, Maroc, Libye et Iran) : essais et guide bibliographique, Paris, Maisonneuve et Larose, 2006.

\section{RÉSUMÉS}

Les premières références à une industrie en Côte d'Ivoire remontent au XVIIe siècle. Elles concernent l'exportation de l'huile de palme produite par des populations autochtones. L'histoire coloniale de la Côte d'Ivoire a débuté par la proclamation de ce territoire des rivières du sud en 
colonie française en 1893. Elle s'achève en 1960 lors de la déclaration des indépendances de plusieurs colonies françaises initiée par le général de Gaulle. Évoquer une industrie en Côte d'Ivoire au regard d'un cadre chronologique qui transgresse la période coloniale suscite des questions. Dans le même ordre d'idées, présenter une industrie postcoloniale et un patrimoine industriel ne sauraient connaître une meilleure appréciation sans des réponses à ces questions. Le fait industriel découle d'un antagonisme entre les techniques introduites par les Européens, colons au départ, coopérants ensuite et enfin partenaires au développement et les pratiques locales des populations de l'espace sylvestre de la Côte d'Ivoire. Cet espace au couvert végétal particulier a été à l'origine d'un choc des savoirs et savoir-faire entre deux peuples, deux cultures et deux approches à la production. La production devenue rapidement rationnelle dans ce qui a été dénommé la «mise en valeur coloniale» a suscité dans un monde devenu tout aussi rapidement marchand avec l'apport d'importants capitaux un développement de modes opératoires, auxquels l'avènement de l'instruction scolaire n'est pas resté sans conséquences. L'école telle qu'introduite sur le territoire a connu un succès important du fait qu'elle ait autorisé une transition qui a permis de construire des secteurs industriels nouveaux basés sur des mutations socio-culturelles et techniques, certes brutales au départ, mais dont l'appropriation a donné naissance à une nouvelle forme d'industrie dont l'artisanat constitue le principal fil conducteur. Des concepts ont été associés à ces formes nouvelles d'industries. Ceux-ci associés à une agriculture de rente, se présentent comme des propositions de réponses à des pratiques agro-industrielles dont l'opportunité de changement s'impose au vu des développements de l'industrie du XXIe siècle. Le patrimoine industriel en est un parfait témoignage et son étude un apport nouveau dans la compréhension de ces territoires.

Prime Industry in Ivory Coast refers to the XVII ${ }^{\text {th }}$ century. It concerns the export of the palm oil produced by native population. The colonial history of Ivory Coast began with the proclamation of South rivers territory in French colony in 1893. Colonisation ends in 1960 during independences statement of several French territories introduced by the general de Gaulle. Industry in Ivory Coast with regard to a chronological frame which breaks the colonial period arouse questions. In the same line, present a post-colonial industry and an industrial heritage would not be well appreciated without answers to these questions. The industrial fact ensues from an antagonism between technology introduced by Europeans (colonists at first, cooperating then and finally partner in the development) and the local practices of populations living in the rain forest area of Ivory Coast. Indeed, this particular space was at the origin of the shock of knowledges between two peoples, two cultures and two approaches in production. Production become quickly rational in what was mentioned the " colonial development ". This fact aroused in the same time of a fast market world including capital. Particularly the school instruction did not stay without consequences. The school introduced in the territory knew an important success because it authorized a transition which has building permit of the new branches of industry based on sociocultural and technical transformations, certainly rough at first, but whose appropriation rise to a new shape of industry taking like small business crafts which constitute the main thread. Concepts were associated to these new forms of industries. These associated to farms, appear as proposals of answers to agro-industrial practices. These also bring the opportunity of change in comparison of the growing industry in the XXI ${ }^{\text {th }}$ century. The industrial heritage is a perfect testimony and its study a new contribution in the understanding of these territories. 
INDEX

Mots-clés : histoire des techniques, agriculture, agro-industrie, savoir traditionnel, patrimoine industriel, forêt

Keywords : history of technology, agriculture, craftsmanship, traditional knowledge, industrial heritage, rain forest, colonial territory

Thèmes : Positions de thèse 